\title{
Identifying and prioritising gaps in colorectal cancer trials research in Australia
}

\section{Rachel F Dea \\ MBBS, PhD, FRACP \\ Senior Lecture \\ Alexandra L Barratt
$\mathrm{MBBS}, \mathrm{MPH}, \mathrm{PhD}$, \\ Professor of Epidemiology \\ Alison Evans \\ $\begin{aligned} \mathrm{PhD}^{2} & \\ \text { Director } & \\ & \end{aligned}$ \\ John Simes \\ $\mathrm{MD}, \mathrm{PhD}$, \\ Director $^{3}$ \\ John Newsom \\ Consumer Representative Scientific Advisory Committee \\ Dan Kent \\ Chair, Consume \\ Advisory Panel ${ }^{4}$ \\ Sally Crossing \\ BEC,
Chair $^{5}$ \\ Catherine Holliday \\ Researcher $^{6}$ \\ Eva Segelov \\ MB BS, FRACP, PhD, \\ Medical Oncologist \\ George Hruby \\ $\mathrm{MBChB}$,
cologist $^{8}$ \\ Radiation Oncologist \\ Martin HN Tattersall \\ Professor of Cancer Medicine}

1 Sydney Medical School, University of Sydney, Sydney,

2 ZEST Health Strategies, Sydney, NSW.

3 National Health and Medical Research Council

Clinical Trials Centre

Sydney, NSW.

4 Australasian

Gastro-Intestinal Trials Group,

Sydney, NSW.

5Cancer Voices NSW.

Sydney, NSW.

6 Cancer Council NSW

Sydney, NSW.

7 St Vincent's Hospital Sydney, NSW.

8 Royal Prince

Alfred Hospital,

Sydney, NSW.

rachel.dear@

sydney.edu.au

MJA 2012; 197: 507-511 doi: 10.5694/mjal2.10623

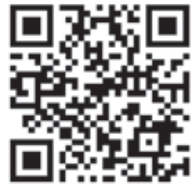

- mija podcast n 2011, we reported a mismatch between the burden of disease and the number of clinical trials in various types of cancer. ${ }^{1}$ For example, colorectal cancer had the highest incidence (14234 cases) and the second highest mortality (4047 deaths) of all cancers in $2007,{ }^{2}$ but only the sixth largest number of cancer trials. ${ }^{1}$ Similarly, poor correlation between burden of disease and public funding of trials has been reported. ${ }^{3,4}$ These mismatches are not limited to cancer. In osteoarthritis, consumers placed a higher priority on research to evaluate knee replacement, education and physical therapy, but $60 \%$ of the studies undertaken evaluated drugs or injections. ${ }^{5}$ Such mismatches may result in evidence "gaps" where the information needed to inform clinical treatment decisions is unavailable. ${ }^{5}$ Further, even when appropriate trials have been conducted, areas of uncertainty or controversy may remain, again contributing to evidence "gaps".

The aims of our study are (i) to identify evidence gaps in the treatment and prevention of colorectal cancer from the perspective of consumers, health professionals, researchers and funding bodies; (ii) to identify trials that could be undertaken to fill those evidence gaps; and (iii) to prioritise clinical trials that could be undertaken by Australian research groups. As part of (iii), we explored the barriers to conducting trials and opportunities to facilitate clinical trials in Australia.

In this study, we focused on colorectal cancer trials and included the perspectives of consumers, health professionals, researchers and funding bodies. We also provided a comprehensive view of priorities for colorectal cancer trials research, allowing differences in perspectives to be examined.

\section{Methods}

The study was conducted jointly by the University of Sydney and the Aus-

Abstract

Objectives: To identify gaps in colorectal cancer clinical trials research in Australia and to suggest and prioritise trials to fill those gaps.

Design, setting and participants: Retrospective review of colorectal cancer trial activity from 1 January 2005 to 1 July 2011 in Australia and internationally, followed by a consensus meeting of consumers, health care professionals, researchers and funding agencies.

Main outcome measures: Proportion of Phase III and randomised clinical trials in the areas of prevention, screening, surgery, adjuvant therapy, advanced disease and behavioural interventions, and priority areas of research identified by participants at the consensus meeting.

Results: The registry search identified 76 colorectal cancer clinical trials (all phases) registered in Australia from 1 January 2005 to 1 July 2011, of which 51 were Phase III or randomised, and 323 Phase III and randomised trials registered worldwide. In Australia, most trials were in advanced colorectal cancer (32), screening (10), and behavioural interventions (9). Worldwide, most Phase III or randomised trials were in advanced disease $(94,29.1 \%)$, surgery $(64,19.8 \%)$, behavioural interventions (38, $11.8 \%)$, and screening (30,9.3\%). At the consensus meeting, all participant groups emphasised the need for research in secondary prevention, screening, individualised treatments and follow-up care after treatment for colorectal cancer.

Conclusions: There is a mismatch between the high proportion of registered trials in advanced colorectal cancer and the areas of priority identified. The development of specific trials in these priority areas depends on the availability of funding and the existence of plausible interventions likely to improve patient outcomes.

tralasian Gastro-Intestinal Trials Group (AGITG), which is Australia's largest independent non-profit organisation conducting clinical trials in the area of gastrointestinal cancers. ${ }^{6}$ The study consisted of two components: a review of colorectal cancer trial activity; and consensus meeting discussions informed by the review.

\section{Review of colorectal cancer trial activity}

We searched the Australian New Zealand Clinical Trials Registry (ANZCTR) and the ClinicalTrials.gov registry for colorectal cancer trials registered from 2005 onwards. We also requested information on trials from the database managers of 12 World Health Organization primary trial registries in Brazil, China, Iran, Japan, Germany, the Netherlands, India, Africa, Cuba, Korea and Sri Lanka, and from the International Standard Randomised Controlled Trial Number Register. We limited the international trials search to
Phase III or randomised trials because these types of trials are most likely to produce results that change clinical practice. We used the following search criteria: the conditions "colorectal" or "bowel" and "cancer"; either open or closed to recruitment; recruiting in all countries; date of registration from 1 January 2005 to 1 July 2011; and Phase III or randomised trials. For colorectal cancer trial activity before 2005, we used a review by O'Dwyer and colleagues, who reported colorectal cancer trial activity up to 31 December 2004 in the categories of risk and prevention, screening, diagnosis and staging, surgery, adjuvant therapy for rectal cancer, adjuvant therapy for colorectal cancer, advanced disease, behavioural interventions (including rehabilitation and lifestyle interventions), novel agents and pharmacogenetics. ${ }^{7}$ We used similar categories to report our findings for the period from 1 January 2005 to 1 July 2011 for comparability. 


\section{Australian colorectal cancer trials registered on ANZCTR and ClinicalTrials.gov} from 1 January 2005 to 1 July 2011

\begin{tabular}{|c|c|c|}
\hline Category and specific research area & $\begin{array}{l}\text { Total trials } \\
\qquad(n=76)\end{array}$ & $\begin{array}{l}\text { Phase III and } \\
\text { randomised } \\
\text { trials }(n=51)\end{array}$ \\
\hline \multicolumn{3}{|l|}{ Prevention } \\
\hline Chemoprevention & 2 & 2 \\
\hline Decision support & 1 & 1 \\
\hline \multicolumn{3}{|l|}{ Screening } \\
\hline New screening methods & 2 & 1 \\
\hline Encouraging participation in screening & 5 & 4 \\
\hline Screening people at high risk of colorectal cancer & 3 & 1 \\
\hline \multicolumn{3}{|l|}{ Role of diagnostic imaging } \\
\hline Preoperative staging of rectal cancer (nodal imaging) & 1 & 0 \\
\hline \multicolumn{3}{|l|}{ Surgery } \\
\hline Biomarkers that predict response to surgery & 1 & 1 \\
\hline Neoadjuvant therapy before surgery for rectal cancer & 1 & 1 \\
\hline Laparoscopic surgery & 5 & 5 \\
\hline Surgery in rectal cancer & 1 & 0 \\
\hline \multicolumn{3}{|l|}{ Adjuvant therapy for rectal cancer } \\
\hline $\begin{array}{l}\text { Better disease staging to identify patients most likely to benefit } \\
\text { from treatment }\end{array}$ & 1 & 1 \\
\hline Optimal systemic therapy regimen to improve overall survival & 1 & 1 \\
\hline \multicolumn{3}{|l|}{ Adjuvant therapy for colorectal cancer } \\
\hline Drugs targeting VEGF and EGFR & 1 & 1 \\
\hline Chemotherapy & 5 & 5 \\
\hline \multicolumn{3}{|l|}{ Advanced disease } \\
\hline Second- and third-line therapies in advanced disease & 6 & 5 \\
\hline Chemotherapy & 3 & 3 \\
\hline Chemoradiation & 4 & 2 \\
\hline Targeted therapies & 6 & 6 \\
\hline Range of new drugs & 13 & 10 \\
\hline \multicolumn{3}{|l|}{ Behavioural interventions* } \\
\hline Quality-of-life interventions & 7 & 1 \\
\hline Survivorship & 2 & 0 \\
\hline \multicolumn{3}{|l|}{ Pharmacogenetics } \\
\hline Genetic markers & 5 & 0 \\
\hline
\end{tabular}

As our purpose was to identify gaps and prioritise trials that could be conducted in Australia, we made an effort to identify all trials that were recruiting or had recruited in Australia through the following:

- a search of the ANZCTR and ClinicalTrials.gov databases, which cover $98 \%$ of all registered clinical trials conducted in Australia ${ }^{1}$ (we used the following search criteria: the conditions "colorectal" or "bowel" and "cancer", either open or closed to recruitment, recruiting in Australia, date of registration from 1 January 2005 to 1 July 2011, all phases);

- the AGITG 2008 Annual Scientific Meeting report, which outlined the feasibility of eight colorectal cancer trials that would recruit patients in Australia; ${ }^{8}$ and topic areas (prevention and screening, and end of life care), which were facilitated by a consumer chairperson, a representative from Cancer Council NSW and an expert in each area, Consumer preferences for research included earlier diagnosis of metastatic disease, the extent of use of best-practice guidelines for palliative care and barriers to change in cancer risk behaviour.

\section{Consensus meeting}

The consensus meeting was held in August 2011 and was run by a professional facilitator. Forty-three people attended, including 14 consumers (people affected by colorectal cancer), 13 health professionals (general practitioners, oncology specialists and nurses), 11 clinical and laboratory researchers, and 5 attendees from non-industry funding bodies with an interest in colorectal cancer.

The meeting began with an overview of the project aims and a summary of our review of colorectal cancer trial activity. This was followed by two 1-hour discussion sessions in which the three groups - consumers, health professionals and researchers - were asked to identify existing gaps and barriers in colorectal cancer trials research. The groups were then asked to propose specific trials that could be done in Australia to fill these gaps, and to identify the barriers to, and opportunities for, implementing these trials. Funding bodies were represented in all three groups.

Each group reported their findings at a plenary feedback session, where all attendees discussed the findings. After the feedback session, a panel that included representatives from each group discussed the proposed trials and challenges. The meeting concluded with a review of the gaps identified.

We obtained ethics approval from the University of Sydney Human Research Ethics Committee (protocol no. 05-2011/13689).

\section{Results} ties across the continuum of cancer using a "global café" process followed by a mailed questionnaire. The global café process involved small group round table discussions under five diagnosis, management, survivorship,

\section{Review of colorectal cancer trial activity}

The search of the ANZCTR and ClinicalTrials.gov registries identified 76 colorectal cancer trials of all phases registered from 1 January 2005 to 1 


\begin{tabular}{|c|c|c|c|c|c|c|c|}
\hline \multirow[b]{2}{*}{ Category } & \multicolumn{6}{|c|}{ Clinical trial registry* } & \multirow[b]{2}{*}{ Total $(n=323)$} \\
\hline & ANZCTR & CT.gov & ISRCTN & ChicTR & RPCEC & IRCT & \\
\hline Prevention & $5(1.5 \%)$ & $9(2.8 \%)$ & $4(1.2 \%)$ & $2(0.6 \%)$ & 0 & $1(0.3 \%)$ & $21(6.5 \%)$ \\
\hline Screening & $7(2.2 \%)$ & $18(5.6 \%)$ & $5(1.5 \%)$ & 0 & 0 & 0 & $30(9.3 \%)$ \\
\hline Preparation for colonoscopy & 0 & $17(5.3 \%)$ & $1(0.3 \%)$ & 0 & 0 & 0 & $18(5.6 \%)$ \\
\hline Role of diagnostic imaging & 0 & $2(0.6 \%)$ & $4(1.2 \%)$ & $1(0.3 \%)$ & 0 & 0 & $7(2.2 \%)$ \\
\hline Surgery & $3(0.9 \%)$ & $38(11.8 \%)$ & $15(4.6 \%)$ & $7(2.2 \%)$ & $1(0.3 \%)$ & 0 & $64(19.8 \%)$ \\
\hline Adjuvant therapy for rectal cancer & $2(0.6 \%)$ & $12(3.7 \%)$ & $1(0.3 \%)$ & $3(0.9 \%)$ & 0 & 0 & $18(5.6 \%)$ \\
\hline Adjuvant therapy for colorectal cancer & $2(0.6 \%)$ & $21(6.5 \%)$ & $3(0.9 \%)$ & $2(0.6 \%)$ & 0 & 0 & $28(8.7 \%)$ \\
\hline Advanced disease & $5(1.5 \%)$ & 76 (24.1\%) & $11(3.4 \%)$ & $2(0.6 \%)$ & 0 & 0 & $94(29.1 \%)$ \\
\hline Behavioural interventions ${ }^{\dagger}$ & $10(3.1 \%)$ & $19(5.9 \%)$ & $7(2.2 \%)$ & $2(0.6 \%)$ & 0 & 0 & $38(11.8 \%)$ \\
\hline Pharmacogenetics & 0 & 0 & $1(0.3 \%)$ & $2(0.6 \%)$ & 0 & 0 & $3(0.9 \%)$ \\
\hline
\end{tabular}

ANZCTR = Australian New Zealand Clinical Trials Registry. ChiCTR = Chinese Clinical Trials Registry. CT.gov = ClinicalTrials.gov. IRCT = Iranian Registry of Clinical Trials. ISRCTN = International Standard Randomised Controlled Trial Number Register. RPCEC = Cuban Public Registry of Clinical Trials.

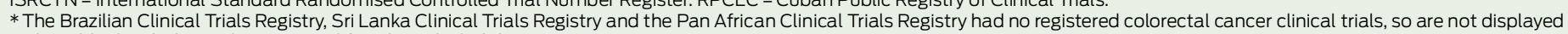
in the table. $†$ Includes trials assessing lifestyle and rehabilitation interventions.

July 2011 that were recruiting (or that had recruited) in Australia (Box 1). Of these, 51 were Phase III or randomised. Most of the trials were in advanced disease (32), screening (10) and behavioural interventions (9).

The search of international registries identified 323 Phase III or randomised colorectal cancer trials (Box 2). Most were in advanced disease (94, $29.1 \%)$, surgery $(64,19.8 \%)$, behavioural interventions $(38,11.8 \%)$ and screening $(30,9.3 \%)$. It is possible that duplicate trials were included in these searches because some trials are registered with more than one clinical trial registry. Data were provided by all trial registries contacted except for those in Germany, the Netherlands, Korea, India and Japan.

\section{Consensus meeting}

The major gaps identified by all three groups in the discussions and trial proposals are shown in Table 1, online at mja.com.au. Many common areas of research were nominated, including secondary prevention in people with adenomas, increasing uptake of the National Bowel Cancer Screening Program, individualised treatment and the long-term effects of treatment on quality of life. Within each of these areas, the details that were discussed differed among the groups. In the category of screening, the health professionals discussed the need for trials of cost-effective methods of screening, while the researchers discussed the importance of using existing research to inform screening intervals. The researchers discussed health services research in the most detail, including the need to identify problems in quality of care in treatment and the importance of conducting studies that meet the needs of the elderly and culturally diverse population.

There were also differences in the perceptions of gaps (see Table 2, online at mja.com.au). Consumers prioritised research questions related to the optimal way of providing information to patients about areas such as disease stage and diet, and the effect of this on patient understanding. They emphasised questions related to quality of life.

In contrast, the health professionals focused on the use of functional imaging in rectal cancer and its use in detecting metastatic disease. They identified a trial to define the role of adjuvant chemotherapy and targeted therapies in Stage III disease according to level of risk.

The researchers discussed trials that could determine the mechanism of resistance to treatments and trials that could identify and test new targets for therapy. They highlighted the benefits of testing new drugs before surgery.

Each group was asked to identify specific trial questions using the "PICO" format (Participant, Intervention, Comparator, Outcome).

The health professionals developed specific clinical trial questions on the primary prevention of colorectal cancer with aspirin, two trials that included strategies to increase the uptake of screening and two trial questions on the adjuvant treatment of rectal cancer. The consumers and researchers produced a list of research questions but did not specify their details during the discussion. It was acknowledged that not all questions may lend themselves to randomised controlled trials, and that other approaches may include research about how to apply existing trial evidence in practice or observational studies.

Conversations about clinical trials inevitably lead to the barriers to conducting the trials. Some of the challenges of conducting the identified trials were difficult to overcome; for example, maintaining the relevance of trial results. However, other issues such as biospecimen collection and funding also present opportunities, including developing a system for tissue banking in Australia and forming collaborative funding initiatives. These and other opportunities for colorectal cancer research in Australia are summarised in Box 3.

\section{Discussion}

Until recently, there was little research about the extent to which the trial questions addressed by researchers matched questions that were important to clinicians and patients. ${ }^{10}$ However, significant progress has been made in this area since 2004 with the establishment of the James Lind Alliance, a not-for-profit independent organisation in the United Kingdom that aims to identify and prioritise research questions of importance to both patients and clinicians. ${ }^{11}$ Through "Priority Setting Partner- 


\section{Barriers to conducting high-quality trials and opportunities in colorectal cancer trials research in Australia}

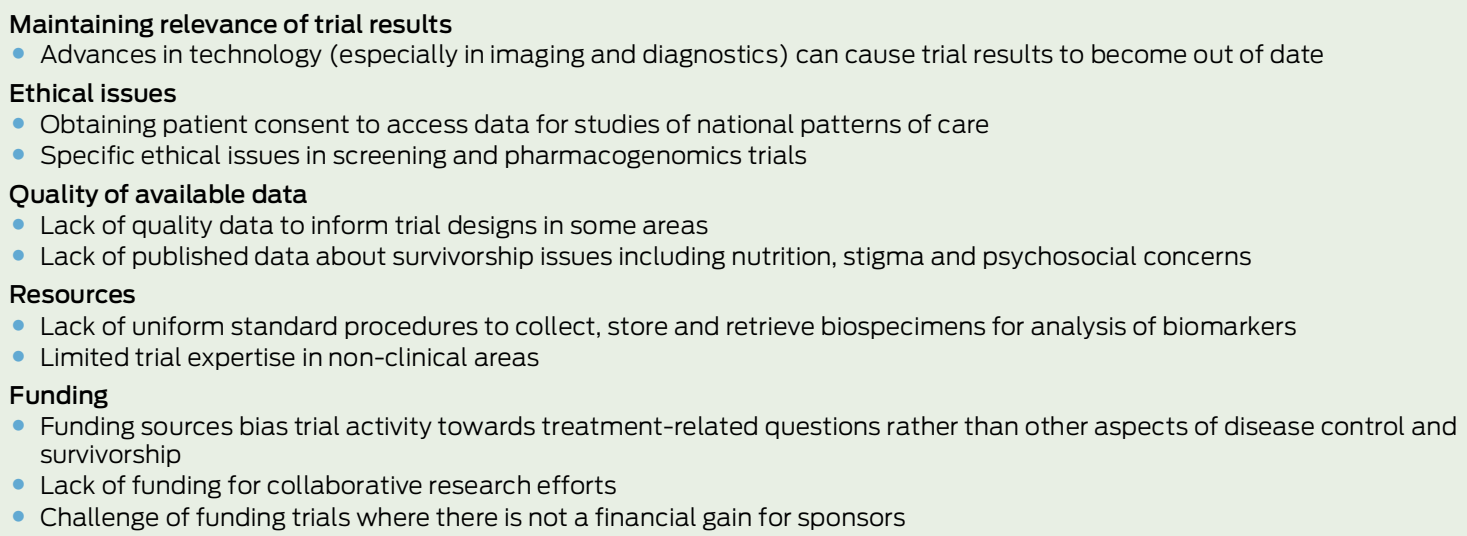

\section{Opportunities}

\section{Biobanking}

- Develop a system for tissue banking in the clinical trial setting (rather than opportunistic collection); this is feasible in Australia

- Applications for research funding may be more attractive if they are from collaborations of groups with disease-specific and research expertise

- Engage gastroenterologists in research

- Lobby for a portion of the Medicare budget to be allocated to research

- Seek opportunities to support clinical research with health economic data; eg, the health economic benefit of increasing participation in screening or that of delivering best-practice care

\section{Quality-of-life outcomesL}

- Consider including quality of life outcomes in all randomised clinical trials (although avoid unnecessary data collection that adds to trial costs)

\section{Data linkage}

- Obtain patient consent to link trial data with databases such as Medicare and the Pharmaceutical Benefits Scheme to increase access to relevant data

\section{Health services research}

- This is a strength for Australia; it is important that the best evidence-based care is put into practice

- In Australia, it is possible to solve problems by implementing government policy change (eg, waiting times for radiotherapy)

- Australia's diverse population provides opportunities to assess the relevance of treatments across different cultural and population groups

ships" they have established a set of guidelines that include quantitative and qualitative methods (including holding focus groups) to incorporate the views of interested groups. ${ }^{12}$ The aim of Priority Setting Partnerships is to produce a "top ten" list of research questions that have the potential to be used by research groups and the pharmaceutical industry. ${ }^{13-16}$

Despite progress in this area, the optimal way to identify and prioritise relevant research is unknown. It is likely that involving research and funding groups from the outset is important. Using clinical trial registry data and obtaining the background research at the start of the process may be a more efficient way to prioritise research questions. Rather than broad research questions, specific clinical trial questions may be more useful for research groups.

To avoid publication bias, we used clinical trial registry data to summa- rise trial activity in colorectal cancer. Despite efforts to increase the rates of registration of clinical trials, registration is not compulsory in Australia, so the results may be incomplete. It is possible that because our search strategy included "colorectal" or "bowel" and "cancer", we may have missed some trials in the area of prevention or those related to the quality of colonoscopy.

The main priority areas identified by $\mathrm{O}^{\prime}$ Dwyer et al included the importance of prevention and screening trials to reduce the burden of colorectal cancer, and the role of individualised treatments. ${ }^{7}$ They did not compare the views of different groups on priority areas for research.

In our study, we used similar categories to O'Dwyer et al so that we could assess the progress made in colorectal cancer trials research since 1 January 2005. A strength of our study is that we explored the views of different groups, recognising that those with an interest in colorectal cancer bring a range of perspectives on priority questions. As reported previously, it is important that research agenda encompass consumer views, so that research findings are relevant to consumer needs. ${ }^{5}$ We made an effort to source consumers with a range of experience and knowledge, although the final group of participants comprised people affected by colorectal cancer. This may have influenced perspectives about screening in the general population. Participating researchers included a pathologist involved in laboratory research, a public health researcher, a psychologist involved in quality-oflife research, four medical oncologists, and a radiation oncologist and a surgeon who were involved as principal investigators of clinical trials. It is likely that the outcomes of our study were influenced by the composition of 
the final group of participants, although the broad representation means that it is likely that the results are generalisable to people with an interest in colorectal cancer research in other countries. Consumers, health professionals and researchers were asked to identify specific trial questions. Although they found this challenging, participants felt that it was more useful to discuss ideas for specific trials that could be developed by research groups, rather than a list of general research areas.

We found a potential mismatch between areas in which trials are being conducted and those prioritised by consumers, health professionals and researchers. The review of trial registry data showed that most trials recruiting patients in Australia and worldwide are testing a range of drugs for use in advanced disease. In contrast, all groups emphasised the need for research in the areas of risk and prevention, screening and survivorship care. A number of specific trials were proposed to fill these gaps. Examples include primary prevention of colorectal cancer with aspirin, and two strategies to increase screening uptake - one in those at aboveaverage risk, and one in healthy adults aged 50 years and over. This is pertinent in the context of the federal government's recent announcement to expand the National Bowel Cancer Screening Program to include people aged 60 years from 2013 and people aged 70 years from 2015. All groups discussed the development of individualised therapies (treatments that are often tested initially in the setting of advanced disease). Other common areas of need that were identified included diagnosis, quality of life and survivorship care (particularly, evaluating the long-term effects of treatment on quality of life and new ways to follow up people affected by color- ectal cancer), and health services research.

There were differences among the topics for future research identified by consumers, health professionals and researchers. For example, the researchers discussed investigating new targeted therapies (eg, stem cells) and understanding mechanisms of resistance to existing treatments in advanced disease, while the consumers discussed whether earlier diagnosis and treatment of metastatic disease would affect outcomes, and how patient expectations can be managed in the setting of advanced disease. Consumers also identified further questions about palliative care, including comparing the experience of dying at home rather in the hospice, and the burden of home death on relatives.

Prevention and screening trials are more difficult to conduct than treatment trials because of the large numbers of patients that need to be recruited, and the high level of funding required. As identified during the consensus meeting, there are opportunities for collaborative research between the AGITG and the Primary Care Collaborative Cancer Clinical Trials Group. The importance of undertaking this research is supported by health economic data, which could help to attract the funding required. The research effort in advanced disease needs to continue, with the goal of individualising patient care to achieve the best outcomes. Routine biobanking will be critical for this to be achieved. The findings from this study could potentially be used by the AGITG and other interested research groups to develop clinical trial protocols.

Acknowledgements: We would like to thank Thuyen Vu and Michael Solomon for their contribution to the study. This study was funded jointly by the National Health and Medical Research Council (Grant No. 512380) and the AGITG. The study sponsors did not contribute to the col- lection of the data, but were responsible for the study design, and had input into the analysis and interpretation of the data, writing of the report, and in the decision to submit the paper for publication.

Competing interests: No relevant disclosures. Received 11 Apr 2012, accepted 15 Aug 2012.

1 Dear RF, Barratt AL, McGeechan K, et al. Landscape of cancer clinical trials in Australia: using trial registries to guide future research. Med J Aust 2011; 194: 387-391. .

2 Australian Institute of Health and Welfare. Cancer. (accessed Aug 2012).

3 Gross CP, Anderson GF, Powe NR. The relation between funding by the National Institutes of Health and the burden of disease. Engl J Med 1999; 340: 1881-1887.

4 Stuckler D, King L, Robinson H, McKee M. WHO's budgetary allocations and burden of disease: a comparative analysis. Lancet 2008; 372: 15631569.

5 Tallon D, Chard J, Dieppe P. Relation between agendas of the research community and the research consumer. Lancet 2000; 355: 2037 2040

6 GI Cancer Institute. Australian Gastrointestinal Trials Group. http://agitg.org.au/ (accessed Mar 2012).

7 O'Dwyer PJ, Eckhardt SG, Haller DG, et al. Priorities in colorectal cancer research: recommendations from the Gastrointestinal Scientific Leadership Council of the Coalition of Cancer Cooperative Groups. J Clin Oncol 2007; 25 2313-2321.

8 Australasian Gastro-Intestinal Trials Group. Minutes of the AGITG colorectal trials workshop; 2008 Sep 5; Sydney, Australia: 1-6.

9 Cancer Council NSW. Consumer research forum; 2009 May 14; Sydney, Australia: 1-14.

10 Oliver S, Gray, J. A bibliography of research reports about patients', clinicians' and researchers' priorities for new research. London: James Lind Alliance, 2006.

11 Cowan K. The James Lind alliance: tackling treatment uncertainties together. J Ambul Care Manage 2010; 33: 241-248.

12 Cowan K, Oliver S. The James Lind Alliance guidebook 2011. http://www.jlaguidebook.org/ (accessed Aug 2012)

13 Thomas RH, Hammond CL, Bodger OG, et al; members of WERN \& James Lind Alliance. Identifying and prioritising epilepsy treatment uncertainties. J Neurol Neurosurg Psychiatry 2010; 81: 918-921.

14 Lloyd K, White J. Democratizing clinical research. Nature 2011; 474: 277-278.

15 Eleftheriadou V, Whitton ME, Gawkrodger DJ, et al. Future research into the treatment of vitiligo: where should our priorities lie? Results of the vitiligo priority setting partnership. Br J Dermato 2011; 164: 530-536.

16 Lophatananon A, Tyndale-Biscoe S, Malcolm E, et al. The James Lind Alliance approach to priority setting for prostate cancer research: an integrative methodology based on patient and clinician participation. BJU Int 2011; 108 : 1040-1043.

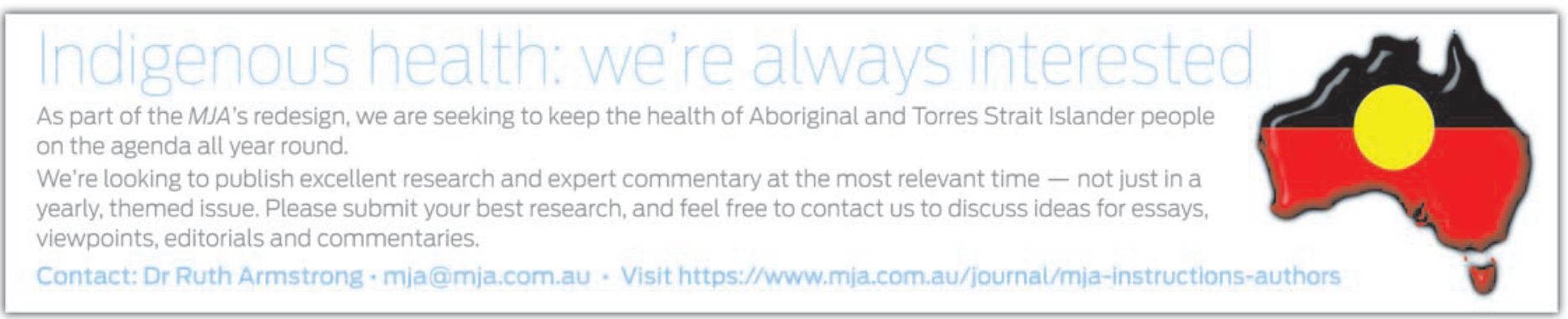

